\title{
Changes of left ventricular function induced by carboplatin combined with paclitaxel in patients with ovarian cancer treated by three-dimensional speckle tracking imaging technology
}

\author{
Zijing Zhai ${ }^{1}$, WenJuan QIN ${ }^{1}$, Zhen Wang ${ }^{1}$, Lei Huang ${ }^{1}$, Shanshan Dong ${ }^{1}$, Xueting Guo ${ }^{1}$, \\ Caiyun Zhang ${ }^{1}$, Zhong Wang ${ }^{1}$, and Guilin $\mathrm{Lu}^{1}$ \\ ${ }^{1}$ Affiliation not available
}

May 12, 2020

\begin{abstract}
Ovarian cancer (OC) is the most common reproductive system tumor threatening women's health. Its incidence rate is increasing year by year. At present, the main treatment of ovarian cancer in the world is the combination of surgery and chemotherapy, and paclitaxel combined with platinum drugs is the first choice for chemotherapy. However, at the same time, studies have shown that platinum and paclitaxel drugs can lead to heart function damage, and serious cases can lead to heart failure. Two dimensional ultrasound and MRI have their own limitations, such as failure to detect lesions early, price and time-consuming, so they are not widely used, and three-dimensional speckle tracking imaging (3D-STE) technology has the advantages of convenience, rapidity, effectiveness and repeatability, which can early detect heart disease. In this paper, 3D-STE technology was used to compare the results of ovarian cancer patients and normal people in different chemotherapy cycles, and compared with the results of MRI. It will be confirmed that 3D-STE technology has more clinical application value than two-dimensional ultrasound and MRI, and provide basis for clinical diagnosis and application.
\end{abstract}

Changes of left ventricular function induced by carboplatin combined with paclitaxel in patients with ovarian cancer treated by three-dimensional spot tracking imaging technology

\#Zijing Zhai ${ }^{1,2}$,\#Wenjuan Qin ${ }^{2}$,\#Zhen Wang ${ }^{1,2}$, Lei Huang ${ }^{2}$, Shanshan Dong ${ }^{2}$, Xueting Guo ${ }^{2}$, Caiyun Zhang ${ }^{2}$, Zhong $\mathrm{Wang}^{3}$, Guilin $\mathrm{Lu}^{2 *}$

1 Medical College of Shihezi University, Shihezi, Xinjiang 832008, China;

2 Department of Ultrasonography, First Affiliated Hospital, School of Medicine, Shihezi University, Shihezi, 832008, China;

3 Department of Cardiology, First Affiliated Hospital, School of Medicine, Shihezi University, Shihezi, 832008, China;

, Shihezi,832008, China

*Address Correspondence to: Guilin Lu; E-mail:690821295@qq.com

\# These three authors contributed equally to this work.

\section{ABSTRAST}

Objective: To investigate the clinical value of three dimensional spot tracking imaging (3D-STE) in detecting left ventricular (LV) systolic function in patients with ovarian cancer (OC) treated with paclitaxel and carboplatin. 
Method: 30 patients with OC were selected and treated with paclitaxel combined with carboplatin chemotherapy. Before chemotherapy, 2-cycle of chemotherapy and 6-cycle of chemotherapy were performed on 2D echocardiography and 3D-STI in each group, and the same examination was performed on 30 normal subjects. Get parameters: left ventricular end diastolic volume (EDV), end systolic volume (ESV), left ventricular ejection fraction (LVEF), stroke output (SV), spherical index (SPI), left ventricular end diastolic mass (LV EDmass), left ventricular end systolic mass (LV ESmass), and left ventricular longitudinal, circumferential, radial and area systolic peak strain (GLS, GCS, GRS, GAS). At the end of chemotherapy, MRI was performed. Statistical analysis was used to compare the above indicators and draw the working characteristic curve of subjects with 3D-STI related parameters, so as to obtain the difference and correlation among groups.

Result: The 3D conventional strain values (LVEF, SV, EDV) of the 2-cycle chemotherapy group and the 6-cycle chemotherapy group were lower than those of the pre chemotherapy group, LV EDmass, LV ESmass increased, left ventricular GLS, GCS, GRS, GAS were significantly lower than those in the pre chemotherapy group, and all had a significant change trend, the difference was statistically significant $(P<0.05)$; There was no significant difference in ESV and EDV between 3D-STI and MRI parameters $(P>0.05)$; 3D-STI and MRI parameters have significant correlation $(P<0.05)$; ROC curve showed that the area under the GAS curve was the highest in the 2-cycle and 6-cycle of chemotherapy for OC. In the 2-cycle of chemotherapy, the highest specificity of GAS was $93.3 \%$, and the highest sensitivity of GLS was $70.0 \%$. In the 6-cycle of chemotherapy, the highest specificity of GAS was $96.7 \%$, and the highest sensitivity of GLS was $96.7 \%$.

Conclusion: The LV systolic function of OC patients treated with paclitaxel and carboplatin was decreased. 3D-STI technology can detect the abnormal LV systolic function of OC patients treated with paclitaxel and carboplatin.

Key words : Three-dimensional spot tracking imaging technology, Ovarian cancer, Carboplatin, Paclitaxel, Left ventricular systolic function

\section{INTRODUCTION}

Ovarian cancer $(\mathrm{OC})$ is the most common reproductive system tumor that seriously threatens women's health ${ }^{[1]}$. At present, the main treatment of OC is the combination of surgery and chemotherapy. The first choice of chemotherapy is paclitaxel combined with platinum drugs ${ }^{[2]}$. Carboplatin is commonly used in platinum drugs, but at the same time, studies have shown that platinum and paclitaxel drugs can cause cardiac dysfunction such as arrhythmia, angina, myocardial infarction, etc, and severe cases can lead to heart failure ${ }^{[3]}$. Echocardiography is a more sensitive method to evaluate the heart damage due to its noninvasive and simple advantages ${ }^{[4]}$. However, due to the lighter heart damage caused by the combination of paclitaxel and carboplatin, it is often easy to ignore the damage, which makes it difficult to accurately assess it early.

The aim of this paper was to explore the clinical application value of 3D-STE in detecting left ventricular (LV) systolic function of patients with OC treated with paclitaxel and carboplatin, so as to provide earlier intervention indexes for clinical practice.

\section{SUBJECTS AND METHODS}

\section{2 .1Research object}

From June 2019 to March 2020, 30 cases of non chemotherapy OC patients were collected from the department of Gynecology, obstetrics and oncology, the First Affiliated Hospital, School of Medicine, Shihezi University. They were germinal epithelial tumors in OC, all of them met the diagnostic criteria of the disease. All of them were treated by carboplatin combined with paclitaxel. The chemotherapy regimen was intravenous administration: TC for 3 weeks, paclitaxel $175 \mathrm{mg} / \mathrm{m}^{2} \mathrm{~d} 1$, carboplatin AUC 5-6d1, a total of 6 courses. Among them: age range: $35-65$ years old, average $(51.6 \pm 7.4)$ years old. At the same time, 30 healthy volunteers in the First Affiliated Hospital of Shihezi University Medical College were selected as the control group, 30 women, $35-65$ years old, average $(48.6 \pm 6.6)$ years old. There was no significant difference in age between the two groups $(P>0.05)$. 


\subsection{Inclusion criteria}

Early diagnosis and treatment of $\mathrm{OC}^{[5]}$.

(1) The patients in the case group were clinically diagnosed as ovarian cancer and had not received chemotherapy (LVEF $>50 \%$ measured by conventional ultrasound). The healthy people in the control group matched with the age, gender, height and weight of the case group (LVEF $>50 \%$ measured by conventional ultrasound);

(2) There were no previous diseases that had affected the structure and function of the ventricle, such as hypertension, diabetes, other autoimmune diseases, myocardial infarction, coronary heart disease, congenital heart disease, heart valve disease, pulmonary heart disease, hypertrophic cardiomyopathy, severe anemia, etc;

(3) Except for pregnant and lactating patients;

(4) Kappa score [?] 70;

(5) All patients signed informed consent.

\subsection{Echocardiography}

All subjects were connected to ECG in the left lying position. The $4 \mathrm{~V}$ probe with the frequency of 1.5-4.0 $\mathrm{MHz}$ of GE Vivid E9 ultrasound system was used to collect the dynamic images of three complete cardiac cycles of each subject in turn, including four chambers, two chambers and three chambers. The images were named in order and stored in the built-in workstation of Echopac 7.0.

3D-STI analysis software is used for post-processing of images. After the two-dimensional images of apical four chambers can be clearly displayed, they are manually converted into $4 \mathrm{D}$ mode. At the same time, patients are instructed to hold their breath and continuously collect: three dynamic and stable cardiac cycles, as well as 3D full volume images. At the same time, the frame rate shall be [?] $40 \%$ of heart rate, and stored and standby, After that, by using 4D auto LVQ software for in-situ analysis, the boundaries of endocardium and epicardium of LV wall were tracked in all frames of the whole cardiac cycle, Then, the parameters were obtained: global longitudinal strain (GLS), global circular strain (GCS), global radial strain (GRS), global area strain (GAS), left ventricular end diastolic volume (EDV), end systolic volume (ESV), left ventricular ejection fraction (LVEF), spherical index (SPI), stroke volume (SV), left ventricular end diastolic mass (LV EDmass), left ventricular end systolic mass (LV ESmass), 17 segment bovine eye map and overall strain time curve of LV myocardium.

\subsection{MRI}

At the end of chemotherapy, the patients were examined by MRI, and the images were collected in the transverse, coronal and sagittal positions, as well as the standard two chamber and four chamber views. The fast gradient echo pulse sequence was used to scan from the bottom of the heart to the tip of the heart, and a total of 6-8 times were collected. The scanning parameters are as follows: repetition time (TR): $39.75 \mathrm{~ms}$, echo time (TE): $1.11 \mathrm{~ms}$, layer thickness: $8 \mathrm{~mm}$, layer spacing: 0mm, field of vision (FOV): 340x276mm, bandwidth: 930hz, and deflection angle: 80deg. After scanning, the epicardial and endocardial regions from the bottom of the heart to the apex of the heart were traced manually after post-processing. After adjustment, the software automatically calculated ESV, EDV and LVEF.

\subsection{Statistical analysis}

SPSS 22 statistical analysis software was used for statistical analysis, and the data conforming to normal distribution was expressed as mean +- standard deviation $(x+-s)$; If it does not obey the normal distribution, it needs to use (median +- quartile spacing) to express, use one-way ANOVA single factor to compare the mean of each group of collected data; In comparison, $L S D-t$ test is needed; ROC curve was used to evaluate the diagnostic efficacy of 3D-STI parameters for paclitaxel combined with carboplatin in the treatment of LV contraction in patients with OC, and the best cutoff point was determined; correlation 
analysis of 3D-STI and MRI parameters based on Pearson correlation analysis; $P<0.05$ was considered significantly different.

\section{Result}

\subsection{Comparison of four groups of 3D conventional ultrasound parameters}

There was no significant difference in the parameters between the pre chemotherapy group and the control group. $(P>0.05)$. However, through the data analysis, we can see that EDV, LVEF, SV decreased, LV EDmass, LV ESmass increased in the 2-cycle and 6-cycle chemotherapy group. (Table 1 and Figure 1)

\subsection{Comparison of systolic strain parameters of 3D-STI in four groups}

There was no significant difference between the pre chemotherapy group and the control group $(P>0.05)$, The GAS, GLS, GCS, GRS of chemotherapy 2-cycle group and chemotherapy 6-cycle group were lower than those of pre chemotherapy group $(P<0.05)$, The results indicate that the whole systolic function of the LV may have been damaged, and with the accumulation of drugs, its 3D strain parameters are gradually reduced. (Table 2 and Figure 2-6)

\subsection{Comparison of conventional parameters of MRI and 3D-STI after chemotherapy}

There was no significant difference in ESV, EDV between the two groups $(P>0.05)$. 3D-STI and MRI parameters have significant correlation $(P<0.05)$. (Table 3 and Figure 7 )

\subsection{ROC curve of LV systolic phase in patients with OC after 3-cycle and 6-cycle of chemother- apy}

The area under the ROC curve of GAS for 2-cycle of chemotherapy is $0.811(P<0.05)$. When the critical value is 34 , the sensitivity and specificity are $93.3 \%$ and $60.0 \%$, respectively, and the Youden index is 0.53 ; The area under the ROC curve of GLS is $0.787(P<0.05)$. When the critical value is 19 , the sensitivity and specificity are $83.3 \%$ and $70.0 \%$, respectively, and the Youden index is 0.53 ; The area under the ROC curve of GCS is $0.756(P<0.05)$. When the critical value is 21 , the sensitivity and specificity are $80.0 \%$ and $56.7 \%$, respectively, and the Youden index is 0.36 ; The area under the ROC curve of GRS is 0.713 ( $P$ $<0.05)$. When the critical value is 53 , the sensitivity and specificity are $90.0 \%$ and $46.7 \%$, respectively, and the Youden index is 0.36 . (Figure $8 \mathrm{~A}$ )

The area under the ROC curve of the 6-cycle GAS of chemotherapy is $0.967(P<0.05)$. When the critical value is 32 , the sensitivity and specificity are $96.7 \%$ and $86.7 \%$, respectively, and the Youden index is at most 0.83; the area under the ROC curve of GLS is $0.944(P<0.05)$. When the critical value is 17 , the sensitivity and specificity are $83.3 \%$ and $96.7 \%$, respectively, and the Youden index is 0.80 ; The area under the ROC curve of GCS is $0.913(P<0.05)$. When the critical value is 19 , the sensitivity and specificity are $83.3 \%$ and $90.0 \%$, respectively, and the Youden index is 0.73 ; The area under the ROC curve of GRS is 0.897 $(P<0.05)$. When the critical value is 50 , the sensitivity and specificity are $90.0 \%$ and $76.7 \%$, respectively, and the Youden index is 0.66.(Figure 8B)

\section{DISCUSSION}

In recent years, epithelial OC has the highest mortality among all gynecological tumors ${ }^{[6]}$. At the same time, the combination of surgery and chemotherapy has gradually become the main treatment of OC. Among them, the combination of paclitaxel and carboplatin can inhibit tumor growth by inhibiting tumor cell division ${ }^{[7]}$. Therefore, it can effectively prolong the survival time of patients with OC. But at the same time, the patients also had different degrees and types of heart damage. The normal function of the heart depends greatly on the LV contraction, while the complex movement of LV in the macro depends on the unique spiral arrangement of myocardial fibers in the micro. Some studies have shown that when the overall function of LV is still in normal state, the change of the local function of LV can provide the early change information of the cardiac function. At the same time, some researches show that 3D-STE can obtain the corresponding 
strain parameters by analyzing the myocardium from multiple directions, so it can better evaluate the change of LV systolic function ${ }^{[8]}$.

SPI, as a new parameter to evaluate the change of LV geometry, had no significant difference between the pre chemotherapy group and the chemotherapy 2-cycle and 6-cycle groups $(P>0.05)$, However, the EDV, LVEF, SV of OC patients in 2-cycle and 6-cycle of chemotherapy showed a trend of progressive decrease compared with those in the pre chemotherapy group, and the LV EDmass and LV ESmass showed a trend of gradual increase, the difference was statistically significant $(P<0.05)$. Therefore, the above parameters can be used as an effective indicator of response to carboplatin combined with paclitaxel chemotherapy for LV damage. At present, MRI is regarded as the gold standard to detect LV systolic function ${ }^{[9]}$. 3D-STI was positively correlated with ESV, EDV and LVEF measured by MRI $(P<0.05)$, and there was no significant difference between the two methods in ESV and EDV results $(P>0.05)$, so 3D-STE is more suitable than MRI in clinical application.

The 3D strain parameters indicate that LV systolic function and structure of the patients after chemotherapy may have changed. However, the peak systolic strain of LV measured by 3D-STE shows that LV has been damaged after chemotherapy with paclitaxel and carboplatin in patients with OC. With the increase of chemotherapy cycle, the overall systolic function of LV gradually decreases, while GLS, GCS, GRS and GAS can reflect the early damage of LV. This result may be due to the use of paclitaxel and carboplatin chemotherapy drugs combined chemotherapy, killing tumor cells at the same time for the normal human tissue cells also have some damage caused by the consequences ${ }^{[7]}$. Especially the damage of myocardial cells, with the increase of chemotherapy cycle, the damage of myocardial cells is aggravating. In clinical, most of the manifestations are the decrease of blood pressure and the increase of heart rate. When the disease is aggravating, it can cause the decrease of myocardial blood supply, myocardial damage, heart failure and even sudden cardiac death. In addition, the experimental results also show that the area under the curve of GAS is the highest, which may be due to the organic combination of GLS and GCS, so the specificity will be higher than the other three strains ${ }^{[10]}$. At the same time, GLS has the highest sensitivity, which may be due to the high sensitivity of the lower endocardial inner myocardium to myocardial damage. The longitudinal inner myocardium of patients with OC undergoing chemotherapy is very vulnerable to be damaged firstly, so the decline of the long axis motor function of the myocardium is the first manifestation. Therefore, it can be concluded that the longitudinal strain of LV is the most sensitive response to the change of LV function ${ }^{[11]}$. Therefore, GAS and GLS can be used as early sensitive indicators to reflect the subclinical state of LV damage.

At present, due to the lack of research on the damage of cardiac function caused by paclitaxel and carboplatin combination chemotherapy, there is still no clear and unified standard for the evaluation of cardiac function damage by 3D strain parameters, which needs further research. At present, carboplatin combined with paclitaxel has a good effect on OC, and has been recognized clinically. However, when LV function is damaged, when the drug dose has a good effect on the tumor, it is necessary to take appropriate drugs to protect the myocardium. In order to maintain the effect of chemotherapy while reducing myocardial damage, or on the basis of ensuring the effect of changing the dosage to reduce organ damage, so as to provide more programs for clinical prevention and treatment.

\section{LIMITATIONS}

High quality image is required by using the 3D-STE technology; Human factors have great influence on ultrasonic image acquisition and post-processing; Small sample size; The age of onset of some patients with ovarian cancer is in the period of greater fluctuation of female hormones, and there are objective factors such as individual differences of patients.

\section{CONCLUSION}

In conclusion, when there is no obvious clinical symptom of cardiovascular system, no change of LV configuration, and all indexes of conventional ultrasound are normal, carboplatin combined with paclitaxel chemotherapy patients may have subclinical myocardial systolic dysfunction, while GLS and GAS have the 
greatest predictive value for myocardial damage. With the advantages of convenience, rapidity, effectiveness and repeatability, 3D-STE technology has shown great potential in the work. It can be more sensitive and comprehensive in the early assessment of left ventricular myocardial damage caused by paclitaxel combined with carboplatin in patients with OC. At the same time, the effective evaluation of LV systolic function can provide a more objective and comprehensive diagnosis and treatment basis for the early clinical intervention of heart damage caused by paclitaxel combined with carboplatin.

\section{REFERENCES}

[1] Odunsi, K. Immunotherapy in ovarian cancer[J]. Annals of Oncology. 2017;28:viii1-viii7.

[2] Ledermann, J A. Front-line therapy of advanced ovarian cancer: new approaches[J]. Annals of Oncology. 2017;28:viii46-viii50.

[3] Uyar D, Bishop E A, Bradley W H, et al. Preliminary data on the use of combination carboplatin, paclitaxel and pembrolizumab therapy for ovarian cancer[J]. Gynecologic Oncology. 2018;149:51-.

[4] Kursat Tigen, Tansu Karaahmet, Cihan Dundar, et al. Yelda Basaran.Right ventricular and atrial functions in patients with nonischemic dilated cardiomyopathy[J]. Wiener Klinische Wochenschrift. 2015;127:877883.

[5] Stephen Lee. Early Diagnosis and Treatment of Cancer: Ovarian Cancer[C]. Acis International Conference on Software Engineering. IEEE Computer Society. 2013;166:234.

[6] Broekman K E, Jalving M, Van T H, et al. Clinical benefit of controversial first line systemic therapies for advanced stage ovarian cancer - ESMO-MCBS scores[J]. Cancer Treatment Reviews. 2018;69:233-242.

[7] Otsuka, Isao. Development and rapid growth of retroperitoneal leiomyoma during carboplatin/paclitaxel chemotherapy in an ovarian cancer patient [J]. European Journal of Obstetrics \& Gynecology \& Reproductive Biology. 2018;231:282-283.

[8] Muraru D, Niero A, Rodriguez-Zanella H , et al. Three-dimensional speckle-tracking echocardiography: benefits and limitations of integrating myocardial mechanics with three-dimensional imaging[J]. Cardiovascular Diagnosis \& Therapy. 2018;8:101-117.

[9] He J, Yang L. Value of three imensional speckle-tracking imaging in detecting left ventricular systolic function in patients with dilated cardiomyopathy[J]. Echocardiography. 2019;36:1492-1495.

[10] Seo Y, Ishizu T, Enomoto Y, et al. Endocardial Surface Area Tracking for Assessment of Regional LV Wall Deformation With 3D Speckle Tracking Imaging[J]. jacc cardiovascular imaging. 2011;4:358-365.

[11] Li Y, Wang Y, Meng X, et al. Assessment of right ventricular longitudinal strain by 2D speckle tracking imaging compared with RV function and hemodynamics in pulmonary hypertension[J]. International Journal of Cardiovascular Imaging. 2017;33:1737-1748. 


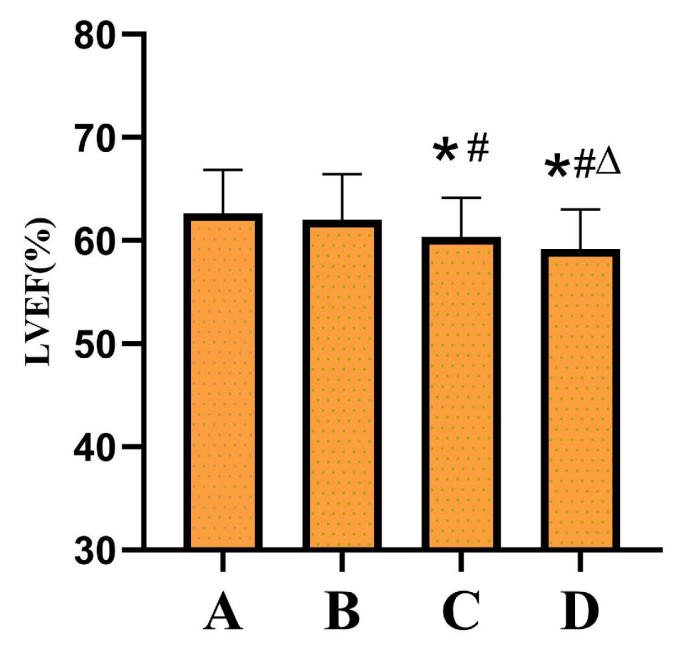

A: Control group B: Pre chemotherapy group C: chemotherapy 2-cycle group D: chemotherapy 6cycle group 

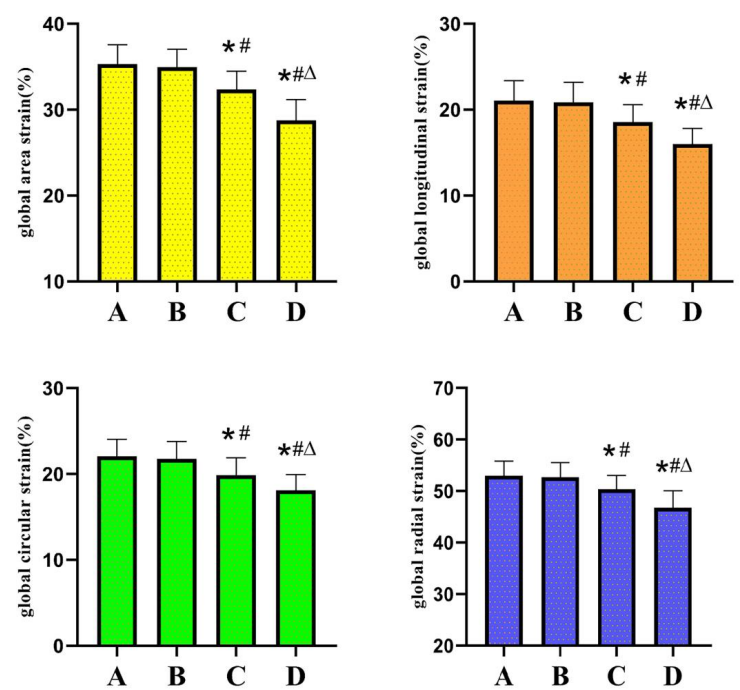

A: Control group B: Pre chemotherapy group C: chemotherapy 2-cycle group D: chemotherapy 6cycle group 


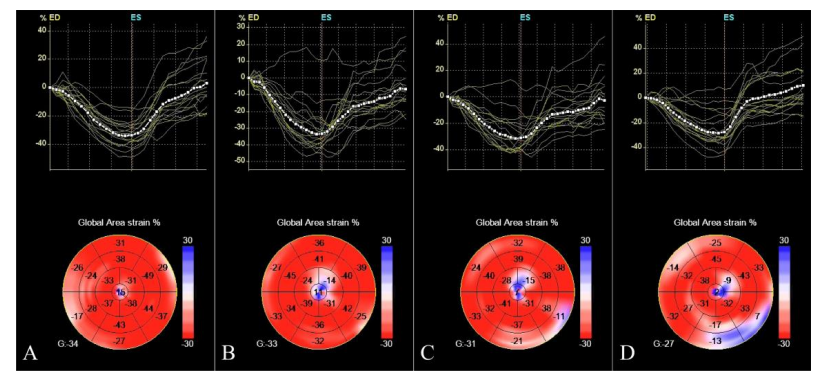

A: Control group B: Pre chemotherapy group C: chemotherapy 2-cycle group D: chemotherapy 6cycle group 


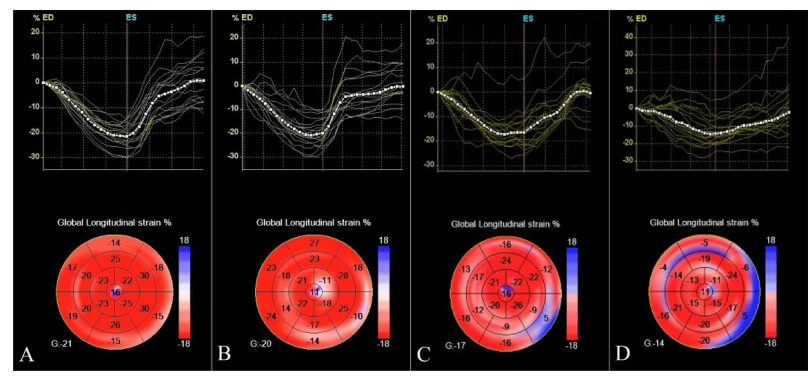

A: Control group B: Pre chemotherapy group C: chemotherapy 2-cycle group D: chemotherapy 6cycle group 


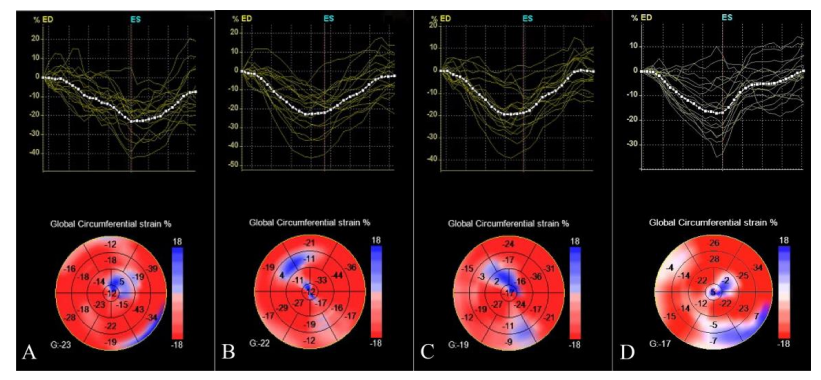

A: Control group B: Pre chemotherapy group C: chemotherapy 2-cycle group D: chemotherapy 6cycle group 


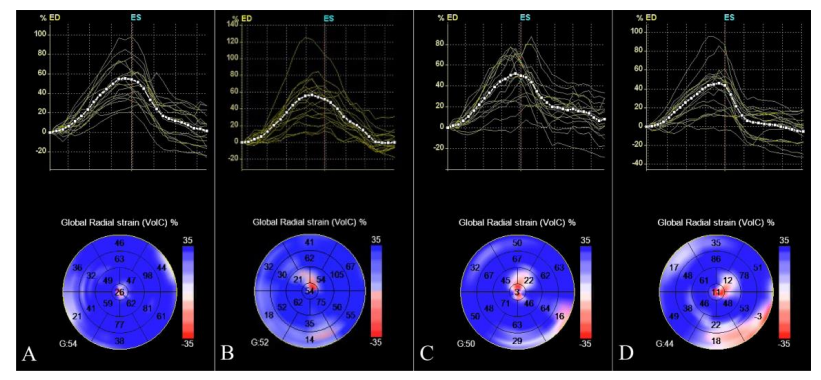

A: Control group B: Pre chemotherapy group C: chemotherapy 2-cycle group D: chemotherapy 6cycle group 


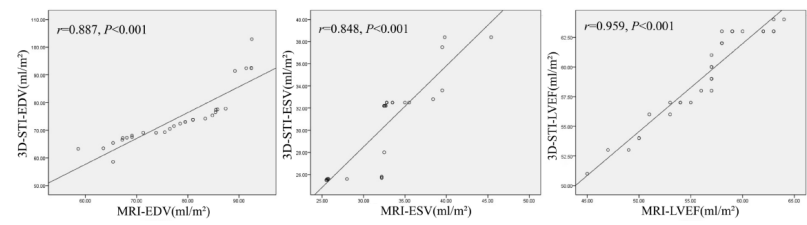



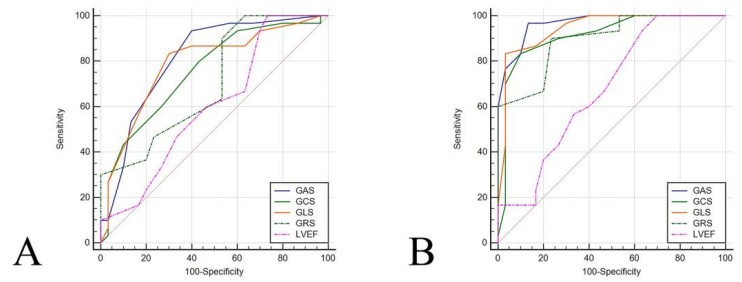

A: chemotherapy 2-cycle group B: chemotherapy 6-cycle group 